\title{
EVALUACIÓN DE UN MÉTODO NUMÉRICO DE MEDICIÓN DEL DIÁMETRO DE LA FIBRA DE ALPACA ${ }^{1}$
}

\author{
Evaluation of a Numeric Method for Alpaca Fibre Diameter Measurement
}

\author{
Haydeé González G. ${ }^{2}$, Carlos León Velarde R. ${ }^{3}$, Raúl Rosadio A. ${ }^{4}$, \\ Wilber García V. ${ }^{5}$ y César Gavidia $C h .^{6}$
}

\section{ReSUMEN}

\begin{abstract}
El presente estudio evalúa el método de medición de diámetro de la fibra de alpaca mediante el procesamiento de imagen digital, Digital Image Fibre Diameter Analisis (DIFDA), desarrollado en el Centro Internacional de la Papa, y lo compara con los valores obtenidos mediante dos métodos de medición convencionales: Lanámetro (Microscopio de Proyección) y OFDA (Optical Fibre Diameter Analysis). El DIFDA requiere de una evaluación del proceso de imágenes digitales obtenidas mediante un escáner de transparencias y negativos de una muestra de fibra de alpaca preparada en porta slides. Este método presenta una opción de procesamiento de imagen digital completa o otra de secciones dentro de la imagen digital, donde los resultados se expresan en promedio, desviación estándar y coeficiente de variación. Un total de de 206 muestras de fibra de alpacas del fundo Pacomarca, Puno, fueron evaluadas. Los valores promedio de diámetro de las fibras fueron de $21.74 \pm 3.03,21.64 \pm 3.58$ y $21.74 \pm 4.01$ según los métodos DIFDA, Lanámetro y OFDA, respectivamente; sin haber diferencia significativa entre promedios. El coeficiente de correlación de Pearson entre DIFDA con el lánametro fue de 0.87 y con OFDA fue de 0.84. La medición del diámetro puede realizarse procesando la imagen digital completa, a partir de tres secciones de 1000 x 1000 píxeles, o desde cinco secciones de 500 x 500 píxeles. Se concluye que no existe diferencia significativa entre los resultados de DIFDA y de los métodos Lanámetro y OFDA, por lo que su uso puede ser de utilidad en programas de mejoramiento animal que requieren medición continua del diámetro de fibras.
\end{abstract}

Palabras clave: fibra de alpaca, diámetro, DIFDA

${ }^{1}$ Estudio financiado por el Proyecto CIP-ALTAGRO

2 Práctica privada. E-mail: haydeegonzalez@gmail.com

${ }^{3}$ Sistemas de Producción y Ambiente, Centro Internacional de la Papa - CIP, Lima. E-mail: c.leonvelarde@cgiar.org

${ }^{4}$ Laboratorio de Microbiología y Parasitología Veterinaria, ${ }^{5}$ Estación Experimental del Centro de Investigación IVITA-Maranganí, ${ }^{6}$ Laboratorio de Medicina Veterinaria Preventiva, Facultad de Medicina Veterinaria, Universidad Nacional Mayor de San Marcos, Lima 
This study presents the statistical evaluation of a digital image alpaca fibre diameter measurement method, Digital Image Fibre Diameter Analysis (DIFDA). This method developed in the International Potato Center (CIP) requires an evaluation of the fibre sample digital image process, prepared in slides covers and taked from a scanner of negatives and films. DIFDA presents two options for fibre diameter measurement process: Digital image complete and sections of the digital image. The results of the fibre sample are mean fibre diameter, standard deviation and coefficient of variability. The objective was to test and compare the mean fibre diameter values obtained by DIFDA with the values from two conventionals methods: Projection Microscope and OFDA (Optical Fibre Diameter Analysis). In adition, DIFDA's process options were evaluated. Two hundred six alpaca fibre samples from Pacomarca Farm, Puno, were evaluated. The mean fibre diameter samples values, measured by commercials methods, Projection Microscope and OFDA, were, $21.64 \pm 3.58$ and $21.74 \pm 4.0$, respectively. The mean fibre diameter reported for DIFDA was $21.74 \pm 3.03$. No statistical differences between the methods were detected. The Pearson's correlation coefficient for DIFDA and Projection Microscope and for DIFDA and OFDA was 0.87 and 0.84, respectively. The process of DIFDA could be done in the complete digital image option, or using three or more sections for the dimention of $1000 \times 1000$ pixels, or five or more sections for the dimention of 500 x 500 pixels. The conclutions were that the DIFDA's results showed no statistical significative differences between neither the Projection Microscope's nor the OFDA's results. Consequently, this measurement method would be used satisfactorily in breeding programs that requires continuos fibre measurement.

Key words: alpaca fibre, diameter, DIFDA

\section{INTRODUCCIÓN}

El Perú es el principal productor de alpacas en el mundo, con una población que representa alrededor del $80 \%$ de la población mundial. La comercialización de la fibra de estos animales representa una actividad económica importante para habitantes de la región alto andina y una alternativa de desarrollo industrial, mediante la exportación de fibra procesada o en textiles (FAO, 2005; ONUDI, 2006). Sin embargo, en el proceso de mejoramiento genético de las alpacas y en la comercialización de su fibra no se hace énfasis en la calidad de fibra que demanda la industria.

El diámetro de la fibra es uno de los factores más importantes en la clasificación de la fibra, el cual podría determinar el precio de la fibra en el mercado. La comercialización generalmente se realiza por peso de vellón; pero hay empresas privadas que otorgan incentivos por finura de vellón. Sin embargo, la medición del diámetro de la fibra representa un problema de costos y de accesibilidad a los métodos existentes, especialmente para los pequeños productores. Algunas muestras son enviadas a laboratorios especializados $\mathrm{y}$, en otros casos, solo cuentan con la inspección visual (Hoffman y Fowler, 1995; Hoffman, 2003; McColl, 2004). Esta deficiencia en la clasificación de la fibra, así como en la identificación de animales productores de fibra fina, representa una debilidad del sistema alpaquero que dificulta aprovechar las oportunidades de desarrollo.

Los animales que poseen fibra fina deben ser considerados como elementos esenciales en programas de selección para el mejoramiento genético (Hoffman y Fowler, 1995; McColl, 2001; Hoffman, 2003). No obstante, no siempre se considera en la selección genética a los parámetros de importancia bio- 
económica, entre los cuales se encuentra el diámetro de la fibra (León-Velarde y Guerrero, 2001).

En la medición de la fibra, el método utilizado debe proporcionar información del diámetro promedio de la muestra (en micras), la desviación estándar y el coeficiente de variación, a fin de ser considerado en planes de mejoramiento genético, así como en la comercialización de animales y de fibra (Hoffman, 2003). Por esto, la División de Sistemas de Producción y Ambiente del Centro Internacional de la Papa (CIP), a través de su proyecto de sistemas de producción, pretende encontrar una solución práctica al problema de medir la finura de la fibra de alpaca en forma cuantitativa, simple y económica. Así, en el CIP se desarrolló un algoritmo matemático (o método numérico), de lectura de imagen digital integrado en un programa de cómputo. Este algoritmo, DIFDA, Digital Image Fiber Diameter Analysis, calcula el diámetro, la desviación estándar y el coeficiente de variabilidad de la fibra.

Por la tanto, en este estudio se evaluó el algoritmo matemático que forma parte del procedimiento mencionado, comparando sus resultados con las mediciones obtenidas por dos métodos de medición convencionales (Lanámetro y OFDA).

\section{Materiales y Métodos}

\section{Generalidades}

Se evaluó el DIFDA con las medidas obtenidas por el Lanámetro y el OFDA (Optical Fibre Diameter Análisis).

La determinación del tamaño muestral (Steel y Torrie, 1960; Little y Hills, 1975; LeónVelarde y Barrera, 2003) fue realizada con la información de 128 muestras analizadas por OFDA, las cuales indicaron un promedio de diámetro de $20.83 \pm 4.94$ micras. Con esta información y considerándose un nivel de confianza de $95 \%$, se calculó un tamaño de 94 muestras de fibra de alpaca; sin embargo, se trabajó con 206 muestras. No se consideró edad, sexo, raza o color de los animales para la colección de las muestras, pero se aseguró la identificación de cada animal.

\section{Muestras de fibra}

Las muestras se tomaron en el Fundo Pacomarca, situado en el distrito de Llalli, provincia de Melgar, departamento de Puno, a una altitud de 4,060 msnm. Las muestras de fibra de alpaca, seleccionadas al azar, correspondían a la región corporal del costillar medio, región que presenta mayor uniformidad respecto al diámetro de la fibra (Aylan-Parker y McGregor, 2001).

La medición del diámetro con OFDA se realizó en el Laboratorio de Control de Calidad de la empresa Inca Tops S.A., Arequipa; la medición mediante Lanámetro se hizo en el Laboratorio de Fibras del Centro de Investigaciones IVITA, Maranganí, de la Universidad Nacional Mayor de San Marcos; y la medición mediante el Algoritmo Matemático (DIFDA) se hizo en la División de Recursos Naturales del Centro Internacional de la Papa, Lima, Perú.

Los resultados de la medición del diámetro mediante OFDA (en forma de histogramas) así como las muestras de fibra fueron proporcionadas por una empresa privada 6 , siendo esta metodología propia de la empresa. La medición del diámetro mediante Lanámetro y DIFDA considera el lavado y secado de muestras.

En la técnica de Lanámetro, se introduce una porción de cada muestra mediante dobleces en el Micrótomo de Hardy. Se hacen cortes a la fibra con una hoja de bisturí,

\footnotetext{
* Inca Tops S.A., Grupo Inka, Arequipa, Perú
} 
(a)

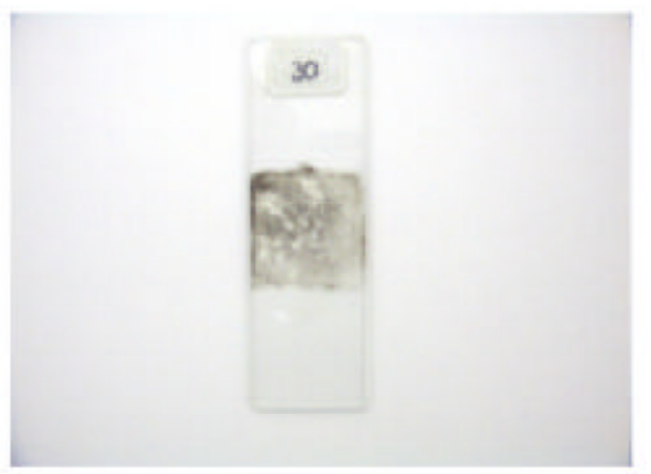

(b)

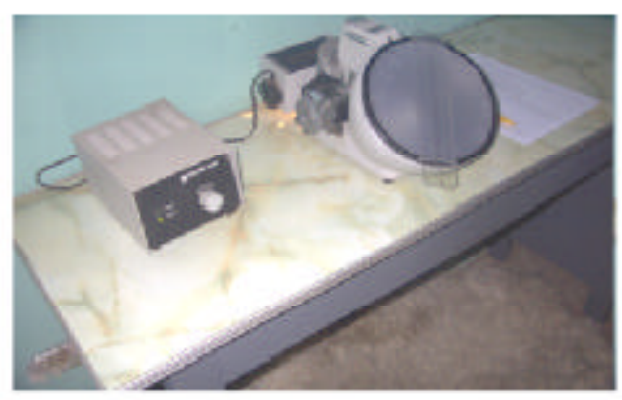

Figura 1. Técnica del Lanámetro. a) Lámina portaobjeto preparada con fibra de alpaca para el proceso de medición de diámetro mediante el Lanámetro. b) Nótese la hoja de registro para las mediciones en el equipo.

(a)

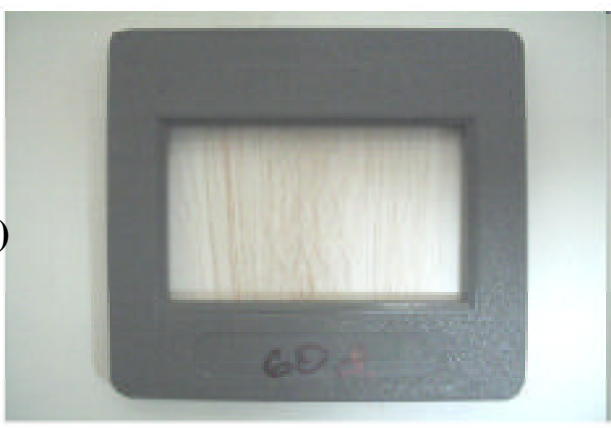

(b)

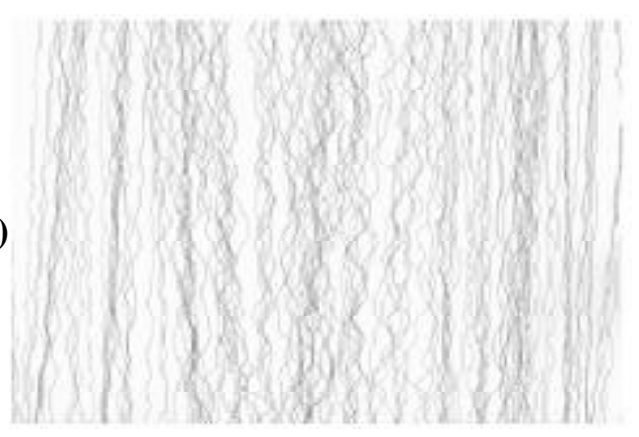

Figura 2. Medición del diámetro de la fibra de alpaca mediante el algoritmo matemático (DIFDA). (a) Porta slide preparado con fibra de alpaca. (b) Imagen digital de una muestra de fibra de alpaca.

dejándolos caer sobre una lámina portaobjeto. Se esparcen con una a tres gotas de Bálsamo de Canadá, y se cubre con una laminilla cubreobjeto (Fig. 1a). Las láminas se colocan en la estufa a $80{ }^{\circ} \mathrm{C}$ por 5 horas. Una vez secadas, se mide el diámetro de las muestras (láminas). Se considera la lectura de 300 segmentos de fibra de cada una de ellas (Fig. 1b).

La medición mediante el Algoritmo Matemático (DIFDA) se realizó mediante procesamiento de imágenes digitales. Estas se obtienen mediante escaneo de porta slides preparados con fibras de alpaca, acomodadas en forma vertical, evitando que se entrecrucen entre ellas (Fig. 2a). Se prepa- raron 1030 porta slides ( 5 por cada muestra de fibra).

El porta slide con la muestra de fibra se escanea mediante un escáner de transparencias y negativos (Nikon Super Coolscan $5000 \mathrm{ED}$ ). La imagen digital se obtiene a una resolución de 4,000 píxeles/pulgada, en la escala de grises, con ancho de 3,946 píxeles y alto de 3,550 píxeles (Fig. 2b). Las imágenes digitales se procesaron con las dos opciones en que podía ser utilizado el algoritmo matemático: Procesamiento de imagen digital completa y procesamiento de secciones cuadradas dentro de la imagen digital. Se realizó pruebas con el número (1-5) de estas seccio- 
Cuadro 1. Características de tres métodos de medición de diámetro de fibra de alpaca

\begin{tabular}{|c|c|c|c|}
\hline & Lanámetro & OFDA & DIFDA \\
\hline $\begin{array}{l}\text { Tiempo de medición } \\
\text { por muestra }\end{array}$ & $30-45 \min$ & $30 \mathrm{~s}$ & $\begin{array}{l}36 \mathrm{~s} \text { (Imagen digital } \\
\text { completa) } \\
1-13 \text { seg (Secciones) }\end{array}$ \\
\hline Equipo & Laboratorio & $\begin{array}{l}\text { Laboratorio } \\
\text { Cómputo }\end{array}$ & Cómputo \\
\hline Materiales & $\begin{array}{l}\text { Láminas portaobjeto } \\
\text { Laminillas cubreobjeto } \\
\text { Bálsamo de Canadá } \\
\text { Micrótomo de Hardy }\end{array}$ & $\begin{array}{l}\text { Guillotina } \\
\text { Láminas de } \\
\text { vidrio }\end{array}$ & $\begin{array}{l}\text { Porta slides } \\
\text { comerciales }\end{array}$ \\
\hline \multirow[t]{2}{*}{ Acceso } & Fabricante & Fabricante & $\begin{array}{l}\text { Equipo comercial } \\
\text { (Escáner) }\end{array}$ \\
\hline & Importador & Importador & Software: Bien Público \\
\hline Inversiones en equipo & $* * * *$ & $* * * * * * * * * *$ & $* *$ \\
\hline
\end{tabular}

* Se considera el valor relativo del Lanámetro como referencia de comparación

nes: 1000 x 1000 y 500 x 500 pix. Al final de cada proceso de medición, se almacenó en forma automática los resultados del algoritmo matemático.

En el Cuadro 1 se presentan las características de los tres métodos empleados en el estudio.

\section{Análisis estadístico}

Se utilizó la prueba estadística de comparación de medias pareadas (T de Student) y la prueba de Correlación Pearson, utilizando el SAS (Statistical Analysis System) (Ref.).

Para determinar el error que podría existir por la variabilidad en la preparación de las muestras se evaluó el cuadrado medio del error mediante Análisis de Varianza que incluyó entre muestras y sub-muestras dentro de muestras.

\section{Resultados y Discusión}

El DIFDA se presenta como un método económico y de fácil uso por el equipo que requiere, el cual es de fácil mantenimiento y transporte.

El Cuadro 2 presenta las mediciones de las muestras de fibra de alpaca, mediante los tres métodos utilizados en este estudio, donde se observa que no existe diferencia significativa entre métodos.

La variabilidad encontrada en el diámetro se debe a la variación que existe entre y dentro de las fibras, debido a que por diversos factores, aún no del todo esclarecidos, estas presentan diferente finura desde la punta hasta el ápice (Carpio, 1991).

El grado de asociación mediante el coeficiente de correlación de Pearson (r) entre el algoritmo matemático (DIFDA) con el 
Cuadro 2. Resultados de las mediciones de diámetro de fibra de alpaca mediante un algoritmo matemático (DIFDA), el Lanámetro y el OFDA

\begin{tabular}{lccccc}
\hline Método & $\mathrm{N}$ & $\begin{array}{c}\text { Promedio } \\
(\mu)\end{array}$ & d.e. & C.V. & I.C. $^{2}$ \\
\hline DIFDA & 206 & 21.74 & 3.03 & $13.94 \%$ & $21.33-22.15$ \\
Lanámetro & 206 & 21.64 & 3.58 & $16.52 \%$ & $21.15-22.13$ \\
OFDA & 206 & 21.74 & 4.00 & $18.38 \%$ & $21.19-22.29$ \\
\hline
\end{tabular}

${ }^{1}$ Coeficiente de variación

2 Intervalo de confianza del 95\%

Cuadro 3. Diferencias de media absolutas $(\mu)$ entre las comparaciones de las mediciones obtenidas mediante las dos opciones del procedimiento de imagen digital DIFDA

\begin{tabular}{|c|c|c|c|c|c|c|c|c|c|c|c|}
\hline & & (A) & & $0 \times 5$ & & & & & $0 \times 10$ & & \\
\hline & & (B) & 2 & 3 & 4 & 5 & 1 & 2 & 3 & 4 & 5 \\
\hline & (B) 1 & $\begin{array}{r}1_{[21.60]} \\
0.04\end{array}$ & $\begin{array}{c}{[21.61]} \\
0.02\end{array}$ & $\begin{array}{c}{[21.61]} \\
0.03\end{array}$ & $\begin{array}{c}{[21.54]} \\
0.10\end{array}$ & $\begin{array}{l}{[21.62]} \\
0.02\end{array}$ & [21.64] & $\begin{array}{l}{[21.56]} \\
0.07\end{array}$ & $\begin{array}{l}{[21.66]} \\
0.03\end{array}$ & $\begin{array}{l}{[21.63]} \\
0.01\end{array}$ & $\begin{array}{l}{[21.67]} \\
0.04\end{array}$ \\
\hline & $\begin{array}{r}{[21.64]} \\
2\end{array}$ & & 0.05 & 0.05 & 0.03 & 0.06 & & & 0.10 & 0.07 & 0.11 \\
\hline 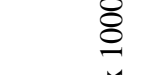 & $\begin{array}{r}{[21.56]} \\
3\end{array}$ & & & 0.05 & 0.13 & 0.04 & & & & 0.03 & 0.01 \\
\hline 8 & $\begin{array}{r}{[21.66]} \\
4\end{array}$ & & & & 0.10 & 0.01 & & & & & 0.04 \\
\hline & $\begin{array}{r}{[21.63]} \\
5\end{array}$ & & & & & 0.05 & & & & & \\
\hline $\mathbb{\Xi}$ & $\begin{array}{r}{[21.67]} \\
1\end{array}$ & & 0.02 & 0.01 & 0.06 & 0.02 & & 0.03 & 0.07 & 0.03 & 0.08 \\
\hline ○ & $\begin{array}{r}{[21.60]} \\
2\end{array}$ & & & 0.00 & 0.08 & 0.01 & & & 0.05 & 0.02 & 0.06 \\
\hline $\begin{array}{l}n \\
\check{x} \\
\varnothing\end{array}$ & $\begin{array}{r}{[21.61]} \\
3\end{array}$ & & & & 0.08 & 0.01 & & & & 0.02 & 0.06 \\
\hline & $\begin{array}{r}{[21.61]} \\
4\end{array}$ & & & & & 0.08 & & & & & 0.14 \\
\hline $\begin{array}{l}\text { Imagen D } \\
\text { Completa }\end{array}$ & $\begin{array}{l}{[21.54]} \\
\text { gital } \\
{[21.74]}\end{array}$ & 0.14 & 0.12 & 0.13 & $0.20^{*}$ & 0.12 & 0.10 & $0.18^{*}$ & 0.08 & 0.11 & 0.06 \\
\hline
\end{tabular}

A: Dimensión (pixeles) de la sección o caja

B: Número de cajas o secciones.

* $(p<0.01)$

Lanámetro fue de 0.87 y con OFDA de 0.84; y entre el Lanámetro y OFDA fue de 0.89 . La variabilidad de muestreo medido por el cuadrado medio del error fue $0.82 \mu$. El error, generalmente, se refiere a la preparación de la muestra, ya que es un error dentro de los grupos formados (Steel y Torrie, 1960; Little y Hills, 1975), donde cada grupo (muestra) estuvo compuesta por cinco imágenes digitales (submuestras). Así mismo, esta variabilidad también está relacionada a la desuniformidad del diámetro dentro de la submuestra (Aylan-Parker y McGregor, 2001; McGregor, 2002). 
En el procesamiento de las muestras para realizar las mediciones mediante la técnica para DIFDA, la preparación de las muestras implica un proceso sencillo; sin embargo, hay que asegurar que las fibras sean colocadas en forma longitudinal evitando cruzamientos entre ellas.

La forma más conveniente de utilizar DIFDA fue mediante la medición de la imagen digital completa; sin embargo, si el tiempo es restringido, se puede usar la opción de medición de secciones o cajas. En el Cuadro 3 se presenta el análisis estadístico de las opciones que ofrece DIFDA. Se evaluaron 55 comparaciones, de las cuales en $2(3.6 \%)$ se presentó una diferencia estadística significativa entre las medidas obtenidas del procesamiento de imagen digital completa con las medidas del procesamiento de cuatro secciones de dimensión $(500$ x 500) y también con las de dos secciones de dimensión (1000 x 1000). Esto es debido a la variabilidad existente entre y dentro de muestras; no obstante, se debe mencionar que la diferencia absoluta encontrada está dada en micras, por lo que las diferencias halladas son mínimas y difíciles de detectar (Steel y Torrie, 1960; Little y Hills, 1975).

\section{Conclusiones}

? El algoritmo matemático, DIFDA, proporciona mediciones estadísticamente iguales a las obtenidas por los métodos convencionales de medición de diámetro de fibra de alpaca: Lanámetro y OFDA.

? Las imágenes digitales pueden ser leídas en su totalidad (imagen digital completa) o en secciones de tres para la dimensión 1000 x 1000 píxeles y de cinco para 500 x 500 píxeles, dependiendo de la cantidad de muestras a medirse.

\section{Agradecimientos}

Los autores agradecen al Proyecto ALTAGRO, coordinado por la División de Sistemas de Producción y Ambiente del Centro Internacional de la Papa, por el apoyo técnico y económico en el desarrollo de este estudio. Especial agradecimiento a la empresa SALLALLI APX, la cual proporcionó las muestras de fibra de alpaca y sus respectivas mediciones por el método OFDA; y al Laboratorio de Fibras del Instituto Veterinario de Investigaciones Tropicales y de Altura (IVITA), Maranganí (FMV-UNMSM), por las facilidades brindadas en las mediciones con el Lanámetro.

\section{Literatura Citada}

1. Aylan-Parker J, McGregor B. 2001 . Optimising sampling techniques and estimating sampling variance of fleece quality attributes in alpacas. Small Ruminant Res 44: 53-64.

2. Carpio M. 1991. La fibra de camélidos. En: Novoa C (eds). Producción de rumiantes menores: Alpacas. Lima: RERUMEN. p 297-359.

3. [FAO] Organización de las Naciones Unidas para la Agricultura y la Alimentación. 2005. Situación actual de los camélidos sudamericanos en Perú. Proyecto de Cooperación Técnica en apoyo a la crianza y aprovechamiento de los Camélidos Sudamericanos en la Región Andina. TCP/RLA/2914.

4. Hoffman E, Fowler ME. 1995. Fiber. In: The alpaca book. USA: Ed. Clay Press. p 44-84.

5. Hoffman E. 2003. Fiber. In: The complete alpaca book. USA: Ed. Bonny Doon. p 235-322.

6. León-Velarde CU, Guerrero J. 2001. Improving quantity and quality of alpaca fibre; using a simulation model for breeding strategies. SAAD III: In Proceeding Tirad International Symposium in Systems Approachs for Agricultural 
Development. SAAD III [CD ROM compatible]. Lima: International Potato Center, CIP. 9 p.

7. León-Velarde C, Barrera V. 2003. Métodos biomatemáticos para el análisis de sistemas agropecuarios en el Ecuador. Quito: Ed. Tecnigrava. $187 \mathrm{p}$.

8. Little T, Hills J. 1975. Statistical methods in agricultural research. University of California. USA. $242 \mathrm{p}$.

9. McColl A. 2001. Understanding and interpreting micron testing. Alpacas are gorgeous. EE.UU. [Internet] [14 marzo 2006].Disponible en:http:// www.alpacasaregeorgeous.com

10. McColl A. 2004. Methods for measuring microns. Alpacas Magazine. Herd Sire 164-168.
11. McGregor A. 2002. Variation in and sampling of alpaca fleeces. State of Victoria, Department of Primary Industries. Australia. [Internet] [09 mayo 2006]. Disponible en: http:// www.dpi.vic.au

12. [ONUDI] Organización de las Naciones Unidas para el Desarrollo Industrial. 2006. Producción textil de fibras de camélidos sudamericanos en el área altoandina de Bolivia, Ecuador y Perú. Informe. Octubre 2006. Subdivisión de Promoción de Inversión y Tecnología. ONUDI. [Internet] [13 mayo 2007]. Disponible en: http:// www.unido.org

13. Steel R, Torrie J. 1960. Principles and procedures of statistics. USA: McGrawHill. 242 p. 